\title{
Cost-effectiveness of nutritional support in the elderly
}

\author{
BY S. P. ALLISON \\ Queen's Medical Centre, Nottingham University Hospital, Nottingham NG7 2UH
}

It is axiomatic that for any treatment to be effective it must be appropriate to the patient's problem. Indiscriminate use of a treatment, in the vague hope that it will do good, is not only ineffective and costly but may do harm. The Veterans' Administration study of peri-operative intravenous feeding (Veterans' Affairs Total Parenteral Nutrition Study Group, 1991), for example, showed a reduction in the complications of surgery among those who were previously undernourished, but a worse outcome and an increase in complication rate among those without significant antecedent malnutrition. What, then, is the clinical problem we are trying to address, and how do we diagnose it? We have evidence from the Irish Republican Army hunger strikers (Allison, 1992), the records of the Warsaw ghetto (Winick, 1979), and other sources that, assuming a normal starting weight, rapid and continuous weight loss is associated with a $30 \%$ chance of death when the body weight has been reduced by between 35 and $40 \%$. Survival beyond $50 \%$ weight loss or a BMI much below 12 is unlikely. Treatment is not just aimed at preventing death, however, since there are clinically significant disturbances of function which start at about $10 \%$ weight loss and become severe above $20 \%$. They include weakness of muscles (Jeejeebhoy, 1988), particularly the respiratory muscles, loss of immune function (Chandra, 1988), poor wound healing (Windsor et al. 1988), impaired thermo-regulation (Fellows et al. 1985), and depression, irritability and increased fatigue (Keys et al. 1950). Above 30\% weight loss there is serious reduction in cardiovascular and gastrointestinal function with oedema, apathy and diarrhoea (Winick, 1979). There is also secondary inhibition of appetite which, curiously, is reversed by enteral feeding techniques (Bastow et al. 1985). All these phenomena are observed regularly in clinical practice and are associated with increased morbidity and complication rate from illness, prolonged hospital stay and an increased chance of dying.

Having defined the degree of undernutrition which is clinically significant, how do we screen the patients for it? One would have thought it a simple matter to measure weight, to take a history of changes in appetite and weight, and to record these in the patient's notes. McWhirter \& Pennington (1994) have shown, however, that we have not even reached the starting line in this respect. They assessed independently the nutritional status of 500 patients admitted to a teaching hospital, including 100 from the Department of Health Care of the Elderly. Of these patients, 200 were classified as undernourished, of whom 130 were severely undernourished, i.e. with a BMI of $<18$. When they examined the patients' records, only $20 \%$ had ever been weighed and less than $50 \%$ had any records relevant to nutritional status. Only ten patients were ever referred to the dietitian and these gained weight whilst in hospital. The majority, who were never referred, lost on average a further $5 \%$ of their body weight during their hospital stay. It seems almost absurd, therefore, to talk about the cost-effectiveness of a treatment when we are not even collecting the information necessary to make the diagnosis. Some centres are addressing this problem, with the introduction of simple, practical nutritional assessment protocols which form part of the medical and nursing admission process. 
QUEEN'S MEDICAL CENTRE NOTTINGHAM, UNIVERSITY HOSPITAL NHS .TRUST NUTRITION ASSESSMENT CHART

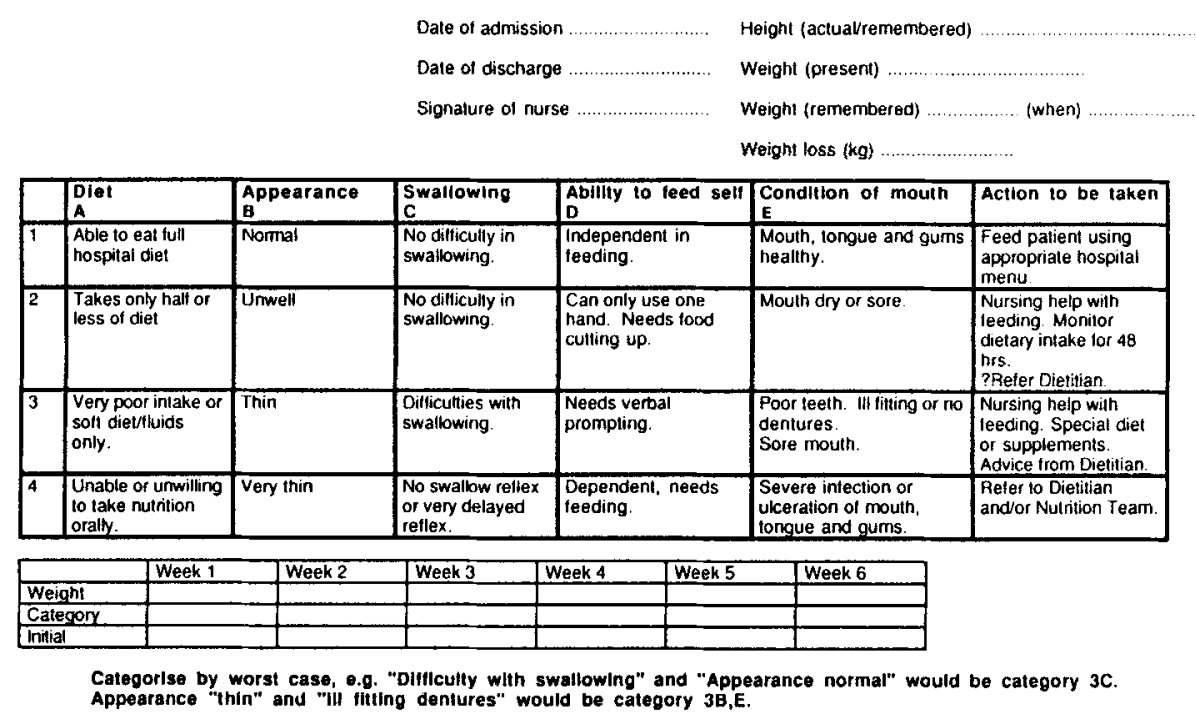

Fig. 1. Nutrition assessment scheme used at Queen's Medical Centre, Nottingham.

We have recently introduced the scheme shown in Fig. 1 for use in our own hospital as part of the nursing admission process. In the past, many schemes of nutritional assessment were over-elaborate, took too long to complete, and were not always appropriate for elderly patients. Scoring systems may also be insufficiently sensitive. Simple qualitative assessment, combined with weight, has been found to be sufficiently valid and sensitive for clinical use (Detsky et al. 1987). Patients at risk can then be assessed in more detail if necessary. Height measurements are problematic in the elderly and a surrogate for height, such as demi-span, may be useful in some patients. A BMI, which is closely related to morbidity, may then be derived. A bedside estimation of arm circumference and fat-fold thickness may be supplemented by formal anthropometric measurements for research purposes, but these are usually unnecessary to daily clinical practice and demand training and skill. This approach was used in a study where we were trying to compare the nutritional status of a healthy and active elderly population in the community with that of elderly medical inpatients and elderly women admitted with fractured femur (Mansell et al. 1990). Decreasing mid-arm circumference and triceps skinfold thickness was seen between the three populations, from the healthy elderly in the community, through the medical patients, to the fractured-femur population. The elderly with fractured femur, therefore, can be perceived as a group at special risk. Using similar methods, a Department of Health Committee on Medical Aspects of Food Policy, (1979) defined 7\% of a sample of the elderly population in the community as suffering from malnutrition, usually associated with mental or physical disease and isolation. There is a need, therefore, for more effective nutritional screening in general practice, as well as among hospital patients, in order to identify groups and individuals at special risk from undernutrition. 
For effective management of patients, however, it is necessary to go further than assessment of nutritional status and to address the original causes of the malnutrition, since correcting these may result in improvement without elaborate nutritional support. Particular problems among the elderly may be depression, dementia, or the side effects of drugs. I am not infrequently asked to provide nutritional support by the enteral or parenteral route, only to find that stopping drugs such as antibiotics results in a natural return of appetite and a resumption of normal meals. Gastrointestinal dysfunction, causing a slow transit time and rise in proximal small-bowel bacterial count, results in both anorexia and malabsorption and is not uncommon among elderly inpatients. Effective management of malnutrition in the elderly, therefore, is not just a nutritional exercise. The malnutrition must be seen in the context of an overall clinical picture.

In the same sense that drugs are ineffective if there is non-compliance, unappetizing food or food supplements poorly presented are unlikely to be consumed and, therefore, are wasteful and of no benefit. One of the major causes of undernutrition in institutions is not the failure to provide food, but to deliver it in a manner appropriate to the particular patients. Since food is now served by orderlies, the nursing staff may remain in ignorance of the patient's poor food intake and fail to provide the necessary assistance. Not infrequently the causes of malnutrition are practical. Putting the meal tray, for example, on the right side of a patient with a right hemiplegia is unlikely to lead to its consumption. Similarly, food that is out of reach may be beyond the effort of the sick or food that is wrapped or inaccessible may be beyond the capacity of the arthritic patient. Taste and smell are often impaired by age, and illness and therefore, one should study and understand the change in eating habits and food preferences among the sick elderly. Many elderly patients, for example, tend to graze, living on small frequent snacks rather than two or three large meals per day. We found that among elderly fractured-femur patients one of the major differences between the well-nourished, who ate well, and the undernourished, whose intake was poor, was the amount eaten between meals, mainly in the form of biscuits, cakes, sweets and fruit. There is a move in hospital catering to set an example of healthy eating, providing food of low energy density and fat content. This may be inappropriate for the undernourished elderly patient, in whom small energydense meals, interspersed with tempting sweet and creamy cakes, may be the best way to meet the patient's metabolic requirements. Attention to these details may be all that is necessary to maintain and improve the nutritional state of most elderly patients, without recourse to more expensive and elaborate means of nutritional support. Slightly more spent on the catering and nursing budget may well be offset by reductions in waste and in the pharmacy budget, as well as by improved outcome.

In the same way that it is important to give the right drug at the right dosage, so the effectiveness of nutritional support may depend on giving the right substrates in the right proportions and in the right amount. Too little may be useless and too much may be toxic. I remember one $30 \mathrm{~kg}$ elderly lady being fed intravenously who became extremely short of breath in the middle of the night, giving rise to a diagnosis of pulmonary embolism. The reason for her problem, however, was that she was receiving a dose of nutrients more appropriate for a $70 \mathrm{~kg}$ man, thus stimulating an increase in metabolic rate and demand for gas exchange. In a patient with impaired lung function this may be lethal.

The ethics of treatment must also be considered. Aggressive nutritional support techniques may be inappropriate in patients in the last weeks of their life or suffering 
from terminal cancer. Early attempts to treat patients suffering from disseminated cancer, for example, were an expensive failure.

\section{EFFECTIVENESS OF TREATMENT}

Having identified the problem and the patients at risk, what treatment is available and what is the evidence for its effectiveness? Ödlund Olin et al. (1994) from Stockholm studied the effectiveness of giving elderly patients meals of high energy density (10.5 MJ) compared with standard hospital meals $(7.0 \mathrm{MJ})$. With the former, there was a $16 \%$ increase in energy intake and a $5 \%$ increase in weight over 3 weeks, which was sustained 6 weeks later. This was associated with improved well-being and mobility, and costs were increased by only $4 \%$.

Larsson et al. (1990) studied 501 long-stay elderly patients for 28 weeks. They screened patients on admission by simple anthropometric and biochemical means into normal and malnourished groups. Each group was then randomized to receive a normal ward diet or one supplemented by a liquid oral feed, giving $1 \mathrm{MJ}$. They confirmed the findings of others that patients who entered hospital well-nourished deteriorated nutritionally during their stay if they received the ward diet only. They also showed a reduced mortality and an increased discharge rate among those receiving supplements. This was most marked among those who were initially malnourished, but was also seen to some extent among the initially well-nourished patients.

In our own study of fractured-femur patients (Bastow et al. 1983) we were able to show increasing mortality with increasing thinness. Also, while well-nourished patients ate well, their thin counterparts ate very poorly. This latter group were entered into a controlled trial of overnight nasogastric-tube feeding $(4 \mathrm{MJ})$, which reduced the rehabilitation time, i.e. the time taken to achieve independent mobility, from 24 to $16 \mathrm{~d}$ with a slight shortening in hospital stay. Delmi et al. (1990) also showed improved outcome following fractured femur with the use of oral supplements.

More elaborate techniques were employed in the Veterans' Administration (Veterans' Affairs Total Parenteral Nutrition Study Group, 1991) and Maastricht (Von Meyenfeldt et al. 1992) studies of peri-operative nutritional support. In the former, nutrition was provided parenterally and in the latter, enterally and parenterally. Both studies showed a reduction in surgical complications with nutritional support, but only in those whose weight loss before surgery exceeded $10 \%$.

There is substantial evidence, therefore, from controlled trials of the effectiveness of different techniques of nutritional support providing that patient selection is appropriate. Some individual examples illustrate some of the issues already mentioned.

\section{CASE HISTORIES}

\section{Case 1}

A 65-year-old woman with severe rheumatoid arthritis had a pre-morbid weight of $69 \mathrm{~kg}$ which had fallen to $45 \mathrm{~kg}$ over 2 years. She underwent surgery to stabilize her neck and post-operatively her BMI had fallen to 15 . She was suffering severe anorexia and, therefore, was referred for enteral or parenteral nutrition. An examination of her treatment chart revealed that she was receiving antibiotics likely to reduce her appetite. These were stopped, her appetite returned and she was able to consume normal meals. The extra costs of artificial nutrition were thereby avoided. 
Case 2

A woman of 73 years old, height $1.55 \mathrm{~m}$, weighed $51 \mathrm{~kg} 2$ years ago. She and her twin sister developed thyrotoxicosis but, whereas her sister lost only $4 \mathrm{~kg}$ in weight, the patient developed depression and anorexia with an associated $35 \%$ weight loss and reduction in BMI from 21.3 to 13.8 . She was apathetic, inactive and fatigued. Nasogastric-tube feeding of $4 \mathrm{MJ}$ daily resulted in an improvement in appetite and an increase in spontaneous mobility, so that after 1 week the enteral-tube feeding could be discontinued and an adequate oral intake was maintained with the assistance of the diet-kitchen cook, who provided small, frequent, tasty snacks. By this time her thyroid function had been reduced to normal by Carbimazole ( $20 \mathrm{mg}$ daily) and she began to gain weight at the rate of $0.5-1 \mathrm{~kg} /$ week. Her oral temperature also rose from a level of $35-35 \cdot 5^{\circ}$ to one of $36 \cdot 5-37^{\circ}$ over a period of 2 weeks, suggesting a gradual restoration of thermogenesis, as well as of appetite and normal mobility.

\section{Case 3}

A woman of 70 years old with motor neurone disease presented with bulbar palsy, so that her oral intake fell to less than 2 MJ daily. She was admitted for insertion of a percutaneous endoscopic gastrostomy, through which she was trained to feed herself at home. She was able to be discharged after $7 \mathrm{~d}$ and maintained herself in a normal nutritional state for the next 18 months, before the inevitable progression of her disease caused her death.

\section{Case 4}

A woman of 78 years old developed complications of surgery for carcinoma of the sigmoid colon with intra-abdominal sepsis, fistula and prolonged gastrointestinal dysfunction. Her BMI preoperatively was only 18 and it was clear by the 7 th post-operative day that she would be unlikely to be able to eat normally for some weeks. Her prognosis, therefore, without nutritional support was considered to be extremely poor. She was treated by parenteral nutrition via a central venous line for a period of 6 weeks until her gastrointestinal dysfunction resolved and she was able to eat normally. It seems unlikely that she would have survived without this form of treatment, which allowed her to make a complete recovery.

\section{NUTRITION TEAMS AND AUDIT}

There is strong evidence that artificial nutrition is most effectively carried out by expert nutrition teams (Allison, 1992; Lennard-Jones, 1992). Central venous feeding, for example, carries an average sepsis rate of $28 \%$ which falls to less than $3 \%$ when conducted by a nutrition team. There is a similar reduction in mechanical complications, as well as improved patient selection and a reduction in costs. Reduced costs stem not only from a reduction in complication rates, but also from standardization and batch processing of feeds, as well as reduction of waste. It has been argued, therefore, that most large district hospitals should have a nutrition team consisting of a nutrition nurse who may be full-time, and a clinician, senior dietitian and pharmacist who spend only part of their time in this work. 
Table 1. Approximate hospital feeding costs $(£)$ per week

\begin{tabular}{llc}
\hline Hospital diet: & Standard & $20 \cdot 00$ \\
& High-energy & $20 \cdot 80$ \\
& With snacks & $22 \cdot 50$ \\
& With oral supplements & $23 \cdot 50$ \\
& $(1 \mathrm{MJ} / \mathrm{d})$ & \\
& Nasogastric or gastrostomy & $70 \cdot 00$ \\
& $(8 \mathrm{MJ} / \mathrm{d})$ & $350-500$ \\
& & \\
Enteral: & & \\
&
\end{tabular}

The fastest growing form of artificial nutrition amongst the elderly is that of home enteral feeding by gastrostomy. This allows early discharge of patients with swallowing problems either to home or to long-stay institutional care. We have recently audited our own experience over 4 years (Hull et al. 1992), mainly in patients with stroke and motor neurone disease. We argued that the initial management and continuing follow-up by an expert member of the nutrition team reduced the severity and frequency of complications and facilitated their effective management if and when they occurred. Emphasis was placed on the use of careful protocols, training of the patients and their families or carers, as well as supportive follow-up.

We have also audited 10 years of experience of in-patient parenteral nutrition (Shields et al. 1994) for prolonged gastrointestinal failure, and assessed the long-term outcome of this treatment. We compared the $50 \%$ in-hospital death rate for acute renal failure managed by dialysis with parenteral feeding for an average period of $50 \mathrm{~d}$ to support patients with acute gastrointestinal failure from non-malignant disease. Life table analysis showed a 10 -year survival rate of $75 \%$ at an average cost of $£ 4700$ per year of life saved. This compares favourably with other well-established medical technologies such as dialysis, renal transplant, breast screening and coronary artery by-pass surgery.

\section{costs}

The approximate costs per week of feeding patients in hospital by the various techniques described previously are listed in Table 1 . The values highlight the enormous difference in cost between simple strategies involving manipulation of oral diet or food supplements on the one hand and the techniques of artificial nutrition on the other. It may be argued, therefore, that a policy designed to identify malnutrition at its early stage and treat it by simple means may prevent some patients deteriorating to the point where more expensive forms of treatment become necessary. The high cost of artificial nutrition, either enterally or parenterally, argues for its use by an expert group within the hospital who will use it in the most appropriate and effective way.

\section{SUMMARY AND CONCLUSION}

There is clear evidence that nutritional support is effective in elderly patients, reducing mortality, morbidity and in some cases hospital stay. Its effectiveness depends upon its being part of an overall management strategy, including screening for patients at risk and appropriate nutritional policies. The extra cost of meals of high energy density, extra 
snacks and food supplements is minimal compared with the potential benefit. Artificial feeding by the enteral or parenteral route is extremely effective, especially when carried out by an expert team and in appropriately-selected patients in whom the outcome justifies the additional expenditure.

\section{REFERENCES}

Allison, S. P. (1992). The uses and limitations of nutritional support. Clinical Nutrition 11, 319-330.

Bastow, M. D., Rawlings, J. \& Allison, S. P. (1983). Benefits of supplementary tube feeding after fractured neck of femur: a randomised controlled trial. British Medical Journal 287, 1589-1592.

Bastow, M. D., Rawlings, J. \& Allison, S. P. (1985). Overnight nasogastric tube feeding. Clinical Nutrition 4, 7-11.

Chandra, R. K. (1988). Immunity and infection. In Nutrition and Metabolism in Patient Care, pp. 598-604 [J. M. Kinney, K. N. Jeejeebhoy, G. L. Hill and O. E. Owen, editors]. Philadelphia: W. B. Saunders.

Delmi, M., Rapin, C.-H., Bengoa, J. M., Delmas, P. D., Vasey, H. \& Bonjour, J. P. (1990). Dietary supplementation in elderly patients with fractured neck of femur. Lancet 335, 1013-1016.

Department of Health Committee on Medical Aspects of Food Policy (1979). Nutrition and Health in Old Age. Report of a Committee on Medical Aspects of Food Policy. London: H.M. Stationery Office.

Detsky, A. S., Baker, J. P., Mendelson, R. A., Wolman, S. L., Wesson, D. E. \& Jeejeebhoy, K. N. (1987). What is subjective global assessment of nutritional status? Journal of Parenteral and Enteral Nutrition 11, 8-13.

Fellows, I. W., Macdonald, I. A., Bennett, T. \& Allison S. P. (1985). The effect of undernutrition on thermoregulation in the elderly. Clinical Science 69, 525-532.

Hull, M. A., Rawlings, J., Murray, F. E. \& Allison, S. P. (1992). An audit of 22 patient years of home enteral nutrition using endoscopic gastrostomy. Clinical Nutrition 11, Special Suppl., 107.

Jeejeebhoy, K. N. (1988). The functional basis of assessment. In Nutrition and Metabolism in Patient Care, pp. 739-751 [J. M. Kinney, K. N. Jeejeebhoy, G. L. Hill and O. E. Owen, editors]. Philadelphia: W. B. Saunders.

Keys, A., Brozek, J. \& Henschel, A. (1950). The Biology of Human Starvation. Minneapolis: University of Minnesota Press.

Larsson, J., Unosson, M., Ek, A. C., Nilsson, L., Thorslund, S. \& Bjurulf, P. (1990). Effect of dietary supplement on nutritional status and clinical outcome in 501 geriatric patients - a randomized study. Clinical Nutrition 9, 179-184.

Lennard-Jones, J. E. (editor) (1992). In A Positive Approach to Nutrition as Treatment. King's Fund Centre Report. London: King's Fund Centre.

McWhirter, J. P. \& Pennington, C. R. (1994). Incidence and recognition of malnutrition in hospital. British Medical Journal 308, 945-948.

Mansell, P. I., Rawlings, J., Allison, S. P., Bendall, M. J., Pearson, M., Bassey, E. J. \& Bastow, M. D. (1990). Low anthropometric indices in elderly females with fractured neck of femur. Clinical Nutrition 9, 190-194.

Ödlund Olin, A., Osterberg, P. \& Hadell, K. (1994). Energy-enriched hospital food to improve energy intake. Journal of Parenteral and Enteral Nutrition 18, Suppl., 31S.

Shields, P. L., Field, J., Rawlings, J., Kendall, J. \& Allison, S. P. (1994). Justification for nutrition teams. Long term survival and cost effectiveness of prolonged in-patient TPN for acute GI failure: 10 years experience. Clinical Nutrition 13, Suppl. 1, 57.

Veterans' Affairs Total Parenteral Nutrition Study Group (1991). Perioperative total parenteral nutrition in surgical patients. New England Journal of Medicine 325, 525-532.

Von Meyenfeldt, M. F., Meijerink, W. J. H. J., Rouflart, M. M. J., Buil-Maassen, M. T. H. J. \& Soeters, P. B. (1992). Perioperative nutritional support: a randomised clinical trial. Clinical Nutrition 11, 180-186.

Windsor, J. A., Knight, G. S. \& Hill, G. L. (1988). Wound healing response in surgical patients: recent food intake is more important than nutritional status. British Journal of Surgery 75, 135-137.

Winick, M. (editor) (1979). In Hunger Disease: Studies by the Jewish Physicians in the Warsaw Ghetto. New York: Wiley. 